\title{
Caracterização dos perfis glicêmicos domiciliares como estratégia para os ajustes insulinoterápicos em pacientes com diabetes mellitus do tipo $1^{*}$
}

\author{
GLYCEMIC PROFILES IN INSULIN ADJUSTMENTS IN PATIENTS WITH TYPE 1 DIABETES MELLITUS \\ CARACTERIZACIÓN DE LOS PERFILES GLICÉMICOS DOMICILIARES COMO ESTRATEGIAPARALOS \\ AJUSTES INSULINO-TERÁPICOS EN PACIENTES CON DIABETES MELLITUS TIPO 1
}

\section{Sonia Aurora Alves Grossi ${ }^{1}$, Tamara Iwanow Cianciarullo², Thaís Della Manna ${ }^{3}$}

$$
\begin{aligned}
& \text { * O presente trabalho } \\
& \text { constitui parte da Tese } \\
& \text { de Doutorado "Avalia- } \\
& \text { ção de dois esquemas } \\
& \text { de monitorização } \\
& \text { domiciliar em pacientes } \\
& \text { com diabetes mellitus } \\
& \text { do tipo 1" apresentada } \\
& \text { à EEUSP no ano de } \\
& \text { 1999. } \\
& 1 \text { Profa. Dra. do Depar- } \\
& \text { tamento de Enferma- } \\
& \text { gem Médico-Cirúrgica } \\
& \text { da Escola de Enferma- } \\
& \text { gem da USP. } \\
& \text { sogrossi@usp.br } \\
& 2 \text { Profa. Titular do Depar- } \\
& \text { tamento de Enferma- } \\
& \text { gem Médico-Cirúrgica } \\
& \text { da Escola de Enferma- } \\
& \text { gem da USP, } \\
& \text { orientadora da Tese de } \\
& \text { Doutorado. } \\
& 3 \text { Mestre em Medicina } \\
& \text { pela Faculdade de } \\
& \text { Medicina da Universi- } \\
& \text { dade de São Paulo, } \\
& \text { Médica Assistente da } \\
& \text { Unidade de Endocri- } \\
& \text { nologia Pediátrica do } \\
& \text { Instituto da Criança do } \\
& \text { Hospital das Clínicas } \\
& \text { da FMUSP. }
\end{aligned}
$$

\begin{abstract}
RESUMO
$O$ artigo tem como objetivo principal caracterizar os perfis glicêmicos domiciliares de pacientes com diabetes mellitus tipo1, a partir de um esquema de monitorização proposto, $e$ adotá-los como estratégia de ajuste nas doses de insulina. Foram realizados 3259 testes, 781 antes do café, 752 antes do almoço, 765 antes do jantar, 740 antes de deitar e 221 pela madrugada. A média das glicemias nestes periodos ultrapassaram os limites superiores satisfatórios em $6,87 \%, 3,83 \%, 11,37 \%$, $30,50 \%$ e $19,28 \%$

respectivamente. Estes dados forneceram subsidios para ajustes nos esquemas insulinoterápicos. Os níveis HbAlc não mudaram de forma significante com os ajustes realizados porém, foram mantidos em $10 \%$.
\end{abstract}

\section{PALAVRAS-CHAVE}

Diabetes mellitus tipol.

Monitorização domiciliar

de glicemia.

Educação do paciente

\section{SUMMARY}

The goal of this paper is to characterize the glycemic profiles of patients with type 1 diabetes mellitus like a strategy in insulin adjustments. A total of 3259 tests were realized being 781 before breakfast, 752 before lunch, 765 before dinner and 740 before bed and 221 in the dawn. The average of the blood glucose tests in these periods oversteped the superior limits in $6,87 \%$, $3,83 \%, 11,37 \%, 30,50 \%$ e 19,28\% respectively. These data gave the conditons to make the insulin adjustments. The HbAlc levels evidenced that there was no significant statistical difference in the metabolic control, but they remained in $10 \%$.

\section{KEYWORDS}

Diabetes mellitus, insulindependent.

Blood glucose self monitoring. Patient education.

\section{RESUMEN}

El objetivo principal de este artículo consiste en caracterizar los perfiles glicémicos domiciliares de los pacientes con diabetes mellitus tipo 1, a partir de un esquema propuesto de monitorización, para aplicarlo como estrategia, en los ajustes de las dosis de insulina. Fueron realizados 3.259 tests, de los cuales 781 se hicieron antes del desayuno, 752 se realizaron antes del almuerzo, 765 antes de la merienda, 740 se hicieron antes de dormir y los 221 se hicieron en la madarugada. La media de las glicemias en estos períodos ultrapasaron los limites superiores satisfactorios em 6,8\%, 3,83\%, 11,37\%, $30,50 \%$ y $19,28 \%$ respectivamente. Estos datos ofrecieron subsidios para poder ajustar los esquemas insulinoterápicos. Los niveles de HbAlc no variaron de forma significativa com los ajustes realizados y se mantuvieron em $10 \%$.

\section{PALABRAS-CLAVE}

Diabetes mellitus, insulinodependiente.Automonitorizacion de la glucose de la sangre.Educacion del paciente. 


\section{INTRODUÇÃO}

A monitorização da glicemia capilar, extremamente enfatizada na atualidade, é considerada um parâmetro importante no manejo do diabetes, pois permite observar os efeitos das atividades e emoções da vida diária nos níveis glicêmicos, direciona a terapêutica, permite ao paciente pequenos ajustes nas doses de insulina e sinaliza as situações de emergência, especialmente as hipoglicemias assintomáticas, favorecendo o processo educativo $^{(1-3)}$. A superioridade dessa monitorização em relação à glicosúria, na viabilidade de obtenção de melhor controle metabólico, é documentada em muitos estu$\operatorname{dos}^{(1,3-5)}$. Entretanto, se a monitorização da glicemia não fizer parte de um plano que implique em maior envolvimento dos pacientes com a equipe ou quando os seus resultados são subtilizados para a adequação terapêutica, não ocorre melhora no controle glicêmico, indicando desta forma que a monitorização por si só não é efetiva ${ }^{(3,4)}$. Evidências a partir de observações clínicas, epidemiológicas e bioquímicas indicam que a manutenção de parâmetros glicêmicos e da pressão arterial próximos à normalidade podem reduzir a incidência e a severidade das complicações neuropáticas, macro e microvasculares ${ }^{(6)}$.

As recomendações do DCCT (Diabetes Control and Complications Trial) ${ }^{(6)}$ como a automonitorização da glicemia capilar 3 a 4 vezes ao dia, múltiplas doses de insulina, alterações nos padrões dietéticos a partir de reeducação alimentar e realização de atividades físicas programadas no sentido de manter os níveis glicêmicos, implicam em mudanças importantes de comportamento dos pacientes. Com relação à automonitorização intensiva da glicemia capilar recomendada pelo DCCT, é preciso levar em consideração que, os altos custos do equipamento e da quantidade de tiras reagentes constituem-se numa limitação importante para a grande maioria dos pacientes. A falta de conscientização sobre a necessidade de melhores controles, a falta de motivação pessoal para uma mudança de comportamento, o desconforto e a dor ocasionados pelo procedimento, o preconceito em assumir a doença perante indivíduos sadios, as frustrações constantes decorrentes de valores glicêmicos inadequados e o desconhecimento sobre como proceder diante dos resultados, são também fatores impeditivos para a realização dos testes ${ }^{(1,7)}$.
Nos últimos anos, muitos questionamentos, reflexões e estudos têm sido realizados a respeito das dificuldades na implementação dos rígidos controles preconizados pelo DCCT e também sobre suas implicações na prática clínica, nos programas educacionais, no autocontrole, na qualidade de vida e no custo do tratamento, especialmente nos serviços que não dispõem de recursos e de profissionais capacitados.

Tem-se observado, por meio da nossa de atuação na Liga de Controle do Diabetes Mellitus do Hospital das Clínicas da Faculdade de Medicina da Universidade de São Paulo(HCFMUSP), junto aos pacientes com diabetes mellitus do tipo1, que existem muitas dificuldades com relação a adesão à monito-rização domiciliar. Embora enfaticamente orientados pela equipe sobre a necessidade e a importância da monitorização para melhor controle metabólico, as limitações individuais para mudança de comportamento e a falta de recursos financeiros para dar suporte ao tratamento, têm sido os fatores impeditivos mais importantes.

Apesar das tentativas de introdução da monitorização sangüínea para todos os pacientes atendidos no nosso serviço, muitos fazem a opção de continuar realizando testes urinários irregularmente ou de não realizar qualquer tipo de controle domiciliar. Desta forma, é bastante freqüente que os ajustes terapêuticos sejam feitos, tendo-se como referência apenas a hemoglobina glicosilada referente ao período e a glicosúria fracionada de 4 períodos, nem sempre adequada, tendo em vista a provável limitação que um único dia de análise urinária pode proporcionar.

As tentativas de intensificação dos controles sangüíneos, nem sempre são bem sucedidas. Embora a monitorização da glicemia capilar seja realizada com facilidade pela maioria das crianças e adolescentes, observa-se que existem dificuldades de adesão, quando mais do que 10 testes semanais são prescritos por longos períodos de tempo. É bastante comum os pacientes realizarem as glicemias em horários diferentes dos preconizados pela equipe ou omitirem a verificação nos horários em que estão fora de casa. Assim sendo, o perfil glicêmico fica irregular e pouco sensível às flutuações glicêmicas, favorecendo a insegurança da equipe para os ajustes terapêuticos principalmente das doses múltiplas de misturas insulínicas.
Caracterização dos perfis glicêmicos domiciliares como estratégia para os ajustes insulinoterápicos em pacientes com diabetes mellitus do tipo 1 
Sonia Aurora Alves Grossi Tamara Iwanow Cianciarullo Thaís Della Manna
Tendo como meta prioritária o melhor controle metabólico possível para os pacientes atendidos em nosso serviço, a equipe tem procurado refletir sobre as estratégias de mudança de comportamento pontuadas e adotá-las na condução do tratamento e nas ações educativas desenvolvidas de forma individual e grupal. Assim sendo, o desenvolvimento de estratégias alternativas de controle do diabetes que vislumbrem as limitações individuais e as deficiências do sistema de saúde relacionadas aos recursos humanos e financeiros são necessárias e constituem-se no objetivo principal deste estudo.

\section{OBJETIVOS}

- Caracterizar os perfís glicêmicos, nos diferentes momentos do dia, a partir da proposição de um esquema diário de monitorização domiciliar da glicemia capilar.

- Avaliar a utilidade dos perfís glicêmicos nos ajustes insulinoterápicos e no controle glicêmico.

\section{CASUÍSTICA E MÉTODO}

O estudo foi realizado na Liga de Controle do Diabetes do HCFMUSP. A autorização prévia para a realização do trabalho consta do parecer sobre o protocolo de pesquisa $\mathrm{n}^{0} .144 / 98$ da Comissão de Ética para Análise de Projetos de Pesquisa da Diretoria Clínica do HCFMUSP.

A população foi constituída de pacientes com diabetes mellitus do tipol que atenderam os seguintes critérios de inclusão: idade não inferior a 2 anos(pela maior vulnerabilidade às infecções respiratórias, as quais elevam os níveis glicêmicos plasmáticos) e condições de realizar monitorização sangüínea $1 \mathrm{vez}$ ao dia pelo período de 6 meses. Os critérios de exclusão da população foram: pacientes com menos de um ano de tempo de doença, aqueles em uso esporádico ou contínuo de medicamentos hiperglice-miantes e pacientes ou cuidadores que não concordaram em participar do estudo.

A amostra foi constituída de 17 pacientes que foram submetidos ao seguinte protocolo: medida da hemoglobina glicosilada (HbAlc) antes do início do estudo, aos 2, 4 e 6 meses após o início do estudo; monitorização e registro domiciliar da glicemia capilar 1 vez ao dia, durante 3 dias consecutivos em cada um dos horários preconizados (antes do café, antes do almoço, antes do jantar e antes de deitar) e quinzenalmente às 3 horas da madrugada, durante o período de 6 meses e retornos quinzenais ao serviço, para participação em encontros educativos, que tinham como objetivos fornecer informações sobre os aspectos importantes no manejo do diabetes, esclarecer dúvidas emergentes e realizar a análise do perfil glicêmico e de sua interação com a dieta, atividades físicas e esquema insulinoterápico e adequação terapêutica.

As dosagens de HbAlc neste estudo foram realizadas pelo método de captura iônica IMx Abbott, tendo como faixa de normalidade os valores entre 5,5 a $8,5 \%{ }^{(8)}$.

Com relação à monitorização da glicemia capilar, os pacientes foram orientados sobre a monitorização domiciliar da glicemia capilar. A determinação da qualidade dos controles das glicemias foi feita tendo-se como referência as recomendações da American Diabetes Association ${ }^{(9,10)}$. Desta forma, os resultados glicêmicos de antes do café da manhã e da madrugada foram considerados bons quando estiveram na faixa compreendida entre 80 - 120 $\mathrm{mg} / \mathrm{dl}$, satisfatórios quando na faixa entre $121-140 \mathrm{mg} / \mathrm{dl}$ e ruins quando menores do que $80 \mathrm{mg} / \mathrm{dl}$ e maiores que $140 \mathrm{mg} / \mathrm{dl}$. Os resultados glicêmicos de antes do almoço e antes do jantar foram considerados bons quando estiveram na faixa entre $80-160 \mathrm{mg} / \mathrm{dl}$, satisfatórios quando na faixa entre $161-180 \mathrm{mg} / \mathrm{dl}$ e ruins quando menores do que $80 \mathrm{mg} / \mathrm{dl}$ e maiores do que $180 \mathrm{mg} / \mathrm{dl}$. Os resultados glicêmicos de antes de deitar foram considerados bons quando compreendidos na faixa entre 101 a $140 \mathrm{mg} / \mathrm{dl}$, satisfatórios quando na faixa entre 141-160 e ruins quando menores do que $100 \mathrm{mg} / \mathrm{dl} \mathrm{e}$ maiores do que $180 \mathrm{mg} / \mathrm{dl}$.

Como parâmetro de controle foi utilizado o controle histórico, de forma que, cada paciente foi controle de si mesmo. Os dados referentes ao esquema insulinoterápico e grau de controle metabólico (por meio de 3 resultados de hemoglobina glicosilada), de todos os pacientes foram analisados retrospectivamente pelo período de 6 a 12 meses anteriores ao início do estudo.

O estudo foi realizado com crianças e adolescentes, juntamente com seus cuidadores (pais ou responsáveis), os quais foram esclarecidos quanto aos objetivos, procedimentos, riscos e benefícios da investigação. $\mathrm{O}$ termo de consentimento pós-informação (Re- 
solução nº.1 de 13/06/1988-CNS), padronizado pela Comissão de Ética do HCFMUSP, foi assinado por todos os pacientes ou seus representantes legais.

Os dados deste estudo foram coletados por meio de 5 instrumentos. Destes, o Instrumento 4 (ANEXO 1), elaborado para este estudo, destinou-se à realização do perfil glicêmico dos últimos 15 dias, a partir dos resultados das glicemias domiciliares realizadas e a coleta de outros dados que se fizeram necessários para os ajustes terapêuticos.

Os resultados estão apresentados em freqüência absoluta e relativa sendo mostrados em forma de tabelas e figuras. As provas estatísticas foram realizadas admitindo-se um erro de primeira espécie de 5\%.

\section{RESULTADOS E DISCUSSÃO}

\section{Caracterização da amostra no momento inicial ao estudo}

A amostra do estudo constituiu-se de 17 pacientes. Destes, $3(17,65 \%)$ encontravamse em idade pré-escolar, $6(35,29 \%)$ em idade escolar, $7(41,17 \%)$ na adolescência e $1(5,89 \%)$ na idade adulta jovem. A faixa etária predominante foi a de adolescentes $(n=7,41,17 \%)$, seguido dos escolares sendo $(\mathrm{n}=6,35,29 \%)$ Em relação ao sexo, o sexo feminino $(64,73 \%)$ foi predominante. Dos constituintes da amostra $82,34 \%$ possuíam diabetes há menos de 9 anos, $11,76 \%$ tinham a doença entre 9 e 12 anos e 5,89\% há mais de 12 anos.

O tratamento insulinoterápico da amostra estudada caracterizou-se pelo uso de insulina de ação intermediária (NPH) usada isoladamente ou associada com insulina de ação rápida $(\mathrm{R})$. As doses variaram de 1 a 2 ao dia, feitas antes do café da manhã (Ac) e antes do jantar (Aj). O uso de insulina de ação ultra rápida (lispro) era feito por apenas 1 paciente, antes do jantar, quando a glicemia estivesse maior do que $180 \mathrm{mg} / \mathrm{dl}$. Este mesmo paciente fazia uso de dose única de NPH-Ac. Pode-se verificar que a maioria dos pacientes (70,59\%)recebiam insulinoterapia de 2 doses diárias e que $41,19 \%$ dos pacientes recebiam associação de insulina de ação intermediária e regular. Portanto 58,81\% dos pacientes, somente utilizavam insulina NPH para o controle metabólico. Com relação à quantidade de insulina administrada por $\mathrm{Kg}$ de peso, $58,82 \%$ dos pacientes tomavam menos de $1 \mathrm{U} / \mathrm{Kg} /$ dia de insulina e $41,18 \%$ tomavam entre 1,01 e $1,5 \mathrm{U} / \mathrm{Kg} / \mathrm{dia}$.

A maioria da amostra encontrava-se eutrófica. Apenas 2 pacientes $(11,76 \%)$ estavam com sobrepeso e $1(5,89 \%)$ com baixo peso. A maioria dos pacientes $(70,59 \%)$ encontrava-se na faixa de $\mathrm{HbA} 1 \mathrm{c}$ compreendida entre 9 e $11 \%$. Ainda, $17,64 \%$ dos pacientes apresentavam-se na faixa entre 7 e $9 \%$ e $11,76 \%$ na faixa entre 11 e $13 \%$. Apenas $1(5,89 \%)$ paciente, apresentava complicações crônicas relacionadas ao diabetes. A presença destas complicações foi evidenciada pelos níveis elevados de microalbuminúria (nefropatia), alteração de sensibilidade em membros inferiores (neuropatia periférica) e gastroparesia (neuropatia autonômica). A paciente em questão tinha 19 anos, diabetes mellitus há mais de 12 anos, $\mathrm{HbAlc}$ de $8,1 \%$ e fazia uso de 2 doses diárias de insulina de ação intermediária.

\section{Perfil das glicemias}

O registro diário da monitorização domiciliar da glicemia capilar, ao longo dos 6 meses de estudo, resultou na realização de 3259 análises sangüíneas. Destas, 781 (23,96\%) foram realizadas antes do café da manhã, 752 $(23,07 \%)$ antes do almoço, $765(23,47 \%)$ antes do jantar, $740(22,70 \%)$ antes de deitar e $221(6,79 \%)$ pela madrugada.

Tabela 1 - Distribuição do total das glicemias realizadas antes do café e madrugada, segundo faixas de valores glicêmicos. São Paulo, 1999

\begin{tabular}{l|c|c|c|c}
\hline \multirow{2}{*}{$\begin{array}{c}\text { VALORES } \\
\text { GLICEMICOS }\end{array}$} & \multicolumn{2}{|c|}{ GLICEMIAS(antes do café) } & \multicolumn{2}{c}{ GLICEMIAS(madrugada) } \\
\cline { 2 - 5 } & $\mathbf{n}$ & $\mathbf{\%}$ & $\mathbf{n}$ & $\%$ \\
\hline$<80$ & 233 & 29,83 & 62 & 28,05 \\
$80-120$ & 151 & 19,34 & 33 & 14,93 \\
$121-140$ & 45 & 5,76 & 7 & 3,17 \\
$141-160$ & 67 & 8,58 & 12 & 5,43 \\
$161-180$ & 37 & 4,74 & 14 & 6,33 \\
$>180$ & 248 & 31,75 & 93 & 42,08 \\
\hline TOTAL & 781 & 100 & 221 & 100,00 \\
\hline
\end{tabular}

Caracterização dos perfis glicêmicos domiciliares como estratégia para os ajustes insulinoterápicos em pacientes com diabetes mellitus do tipo 1

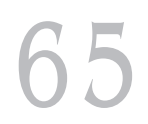

Rev Esc Enferm USP 2003; 37(1): 62-71. 
Sonia Aurora Alves Grossi Tamara Iwanow Cianciarullo Thaís Della Manna
Como pode ser observado na tabela 1 , do total das análises realizadas antes do café da manhã (781), 74,90\% delas foram ruins, 29,83\% por estarem menores do que $80 \mathrm{mg} / \mathrm{dl} \mathrm{e}$ $45,07 \%$ por estarem maiores do que $140 \mathrm{mg} / \mathrm{dl}$. Somente $19,34 \%$ do total destas análises foram boas e 5,76\% satisfatórias.

As hiperglicemias de jejum no paciente com diabetes mellitus podem ser conseqüentes às hipoglicemias noturnas. Isto é particularmente confirmado quando estas hiperglicemias de jejum pioram com o aumento nas doses de insulina de ação intermediária realizadas antes do jantar ou antes de deitar. Este fato, denominado fenômeno de Somogyi, acontece porque a hipoglicemia na madrugada, induzida pela insulinoterapia da tarde ou noite, ativa o processo de glicogenólise hepática e aumenta a secreção dos hormônios contrarreguladores durante à noite, elevando os níveis de glicose sangüínea pela manhã $\tilde{a}^{(11-13)}$.

Por outro lado, as hiperglicemias de jejum, também podem acontecer devido ao decréscimo da ação da insulina, que foi aplicada no dia anterior, nas primeiras horas da manhã seguinte ${ }^{(10)}$. O fenômeno do alvorecer também pode ser o motivo dos aumentos glicêmicos de jejum. Tanto em indivíduos normais, como em diabéticos, a produção hepática de glicose aumenta e a sensibilidade à insulina diminui, entre 4 e 8 horas da manhã. Desta forma, maiores necessidades de insulina existem neste horário para a manutenção da normoglicemia. Além disso, os picos fisiológicos de hormônio de crescimento também acontecem na madrugada, independentemente das respostas contrarreguladoras das hipoglicemias. Assim sendo, o pico de ação da insulina de ação intermediária pode preceder o período no qual as necessidades de insulina são maiores ocasionando hiperglicemias matutinas ${ }^{(11-13)}$. As hiperglicemias de jejum observadas, na maioria dos pacientes deste grupo, podem encontrar explicações nos três processos descritos anteriormente. Muitos pacientes apresentaram hipoglicemias noturnas ( $3 \mathrm{hs}$ da madrugada), seguidas de hiperglicemias de jejum, sugerindo a ocorrência do fenômeno Somogyi. Outros apresentaram hiperglicemias pela madrugada associadas a hiperglicemias de jejum, sugerindo a ocorrência do fenômeno do alvorecer ou o decréscimo da ação da insulina. Tendo em vista que todos os pacientes deste grupo, que faziam uso de 2 doses de insulina, tomavam a segunda dose de insulina de ação intermediária antes do jantar, é muito provável que houvesse redução das hiperglicemias de jejum e das hipoglicemias noturnas se a segunda dose de insulina de ação intermediária fosse realizada antes de deitar pois, desta forma, o pico de ação da insulina seria coincidente com o período de maior necessidade da mesma. Na vigência de hipoglicemias noturnas associadas à hiperglicemias de jejum não se pode deixar de considerar também a necessidade de redução nas quantidades de insulina vespertina. Por outro lado, os que faziam uso de dose única de insulina poderiam ser beneficiados com o fracionamento da dose de insulina de ação intermediária da manhã ${ }^{(12-14)}$.

A tabela 1 mostra ainda que, do total das análises realizadas pela madrugada (221), apenas $14,93 \%$ foram boas e $3,17 \%$ foram satisfatórias. A maioria dos resultados foi ruim, $28,05 \%$ por estarem menores do que $80 \mathrm{mg} / \mathrm{dl}$ e $53,84 \%$ por estarem maiores que $140 \mathrm{mg} / \mathrm{dl}$. Estes resultados reforçam a idéia de que a grande ocorrência das hiperglicemias de jejum, mostradas anteriormente, estejam mais relacionadas ao fenômeno do alvorecer e ao decréscimo da ação da insulina durante à noite ou ainda, a uma possível hipoinsulinização pós prandial ao jantar. Diante deste perfil glicêmico da madrugada pode-se dizer que o fracionamento da dose para os que tomavam uma única aplicação de insulina de ação intermediária e a mudança de horário da segunda dose da insulina da ação intermediária, dos que tomavam 2 doses, para antes de deitar poderia ajudar na reversão destes descontroles glicêmicos. Nos casos de hipoinsulinização pós prandial ao jantar estaria indicado o uso ou a adequação da insulina regular antes do jantar. Ainda assim, não se pode deixar de considerar a possibilidade da ocorrência do fenômeno de Somogyi, tendo em vista a ocorrência de $28,05 \%$ de hipoglicemias pela madrugada e, nestes casos, reduções nas quantidades de insulina da tarde ou noite devem ser pensadas.

A tabela 2 mostra que do total das glicemias realizadas antes do almoço (752), 28,59\% foram boas e 4,39\% satisfatórias. A maioria delas foi ruim, sendo $19,28 \%$ por apresentarem valores menores do que $80 \mathrm{mg} / \mathrm{dl} \mathrm{e} 47,74 \%$ por apresentarem valores maiores do que $180 \mathrm{mg} / \mathrm{dl}$. 
Tabela 2 - Distribuição do total das glicemias realizadas antes do almoço e antes do jantar, segundo faixas de valores glicêmicos. São Paulo, 1999

\begin{tabular}{l|c|c|c|c}
\hline \multirow{2}{*}{$\begin{array}{c}\text { VALORES } \\
\text { GLICEMMICOS }\end{array}$} & \multicolumn{2}{|c|}{$\begin{array}{c}\text { GLICEMIAS(antes do } \\
\text { almoço) }\end{array}$} & \multicolumn{2}{c}{$\begin{array}{c}\text { GLICEMIAS(antes do } \\
\text { jantar) }\end{array}$} \\
\cline { 2 - 5 } & $\mathbf{n}$ & $\%$ & $\mathbf{n}$ & $\%$ \\
\hline$<80$ & 145 & 19,28 & 149 & 19,48 \\
$80-160$ & 215 & 28,59 & 196 & 25,62 \\
$161-180$ & 33 & 4,39 & 44 & 5,75 \\
$>180$ & 359 & 47,74 & 376 & 49,15 \\
\hline TOTAL & 752 & 100 & 765 & 100 \\
\hline
\end{tabular}

As hipoglicemias de antes do almoço poderiam estar relacionadas à inadequação entre a dose de insulina regular administrada antes do café da manhã, a ingestão alimentar e as atividades físicas realizadas no período compreendido entre o café da manhã até o almoço, nos pacientes que faziam uso deste tipo de insulina (41,19\%). Naqueles que não faziam uso de insulina de ação rápida antes do café da manhã (58,81\%), as hipoglicemias pré-almoço poderiam estar relacionadas ao excesso de atividade física ou deficiência na ingestão alimentar no período da manhã ${ }^{(12)}$.

As hiperglicemias de antes do almoço naqueles que não faziam uso de insulina regular pela manhã poderiam ser justificadas pela inatividade física do período ou pela falta de insulina necessária para diminuir os níveis glicêmicos, elevados pela ingestão alimentar matutina, tendo em vista que a insulina de ação intermediária estaria fazendo seu pico de ação, após o almoço. Naqueles que faziam uso de insulina regular pela manhã é provável que estivesse acontecendo uma inadequação entre a insulinoterapia de ação rápida, a atividade física e a ingestão alimentar ${ }^{(15)}$.

O perfil glicêmico dos pacientes referentes ao período antes do jantar, foi semelhante ao do apresentado pelos pacientes antes do almoço, como pode ser observado também na tabela 2. Das 765 glicemias realizadas antes do jantar, $25,62 \%$ foram boas e $5,75 \%$ foram satisfatórias. A maioria delas foi ruim considerando que os pacientes apresentaram 19,48\% de hipoglicemias e $49,15 \%$ de hiperglicemias.

As hipoglicemias neste horário geralmente estão relacionadas ao pico de ação da insulina de ação intermediária administrada pela manhã sem a ingestão alimentar suficiente e/ ou excesso de atividade física ${ }^{(12,14)}$.

Por outro lado, quando as doses de insulina de ação intermediária da manhã são insuficientes, para a redução dos níveis glicêmicos, elevados pela ingestão alimentar do almoço e da tarde, ocorrem as hiperglicemias. A inatividade física à tarde também contribui para as elevações glicêmicas antes do jantar ${ }^{(15)}$.

O perfil glicêmico de antes de deitar, como pode ser observado na tabela 3 evidencia que somente $19,05 \%$ do total dos resultados glicêmicos (740) estavam bons e satisfatórios. A maioria $(80,95 \%)$ apresentou resultados ruins sendo, $59,45 \%$ por se apresentarem com valores acima de $160 \mathrm{mg} / \mathrm{dl} \mathrm{e} 21,48 \%$ por se apresentarem menores que $100 \mathrm{mg} / \mathrm{dl}$.

As hipoglicemias neste horário são potencialmente perigosas pois, podem se agravar durante o período de sono. Elas geralmente refletem a interação entre o pico de ação da insulina regular feita antes do jantar, a ingestão alimentare a atividade física, realizadas neste período, nos pacientes que utilizavam a insulina de ação rápida. Naqueles que não a utilizavam as hipoglicemias podem ter sido originadas por ingestão insuficiente, excesso de atividade física, ou até mesmo pela alteração do pico de ação da insulina intermediária administrada pela manhă ${ }^{(12,14)}$.

As hiperglicemias identificadas antes do deitar, nos pacientes que tomavam a insulina regular antes do jantar, evidenciam geralmente inadequação entre a dose da insulina, ingestão alimentare a atividade física realizadas neste período. Os pacientes que não faziam uso de insulina de ação rápida antes do jantar provavelmente encontravam-se hipoinsuli-nêmicos no período ${ }^{(12,14)}$.

Tabela 3 - Distribuição do total das glicemias realizadas antes de deitar, segundo faixas de valores glicêmicos. São Paulo, 1999

\begin{tabular}{l|c|c}
\hline \multirow{2}{*}{$\begin{array}{c}\text { VALORES } \\
\text { GLICÊMICOS }\end{array}$} & \multicolumn{2}{c}{$\begin{array}{c}\text { GLICEMIAS } \\
\text { (antes de deitar) }\end{array}$} \\
\cline { 2 - 3 } & $\mathbf{n}$ & $\%$ \\
\hline$<100$ & 159 & 21,48 \\
$101-140$ & 102 & 13,78 \\
$141-160$ & 39 & 5,27 \\
$161-180$ & 45 & 6,08 \\
$>180$ & 395 & 53,37 \\
\hline TOTAL & 740 & 100,00 \\
\hline
\end{tabular}

Caracterização dos perfis glicêmicos domiciliares como estratégia para os ajustes insulinoterápicos em pacientes com diabetes mellitus do tipo 1 
Sonia Aurora Alves Gro Tamara Iwanow Ciancial Thaís Della Manna

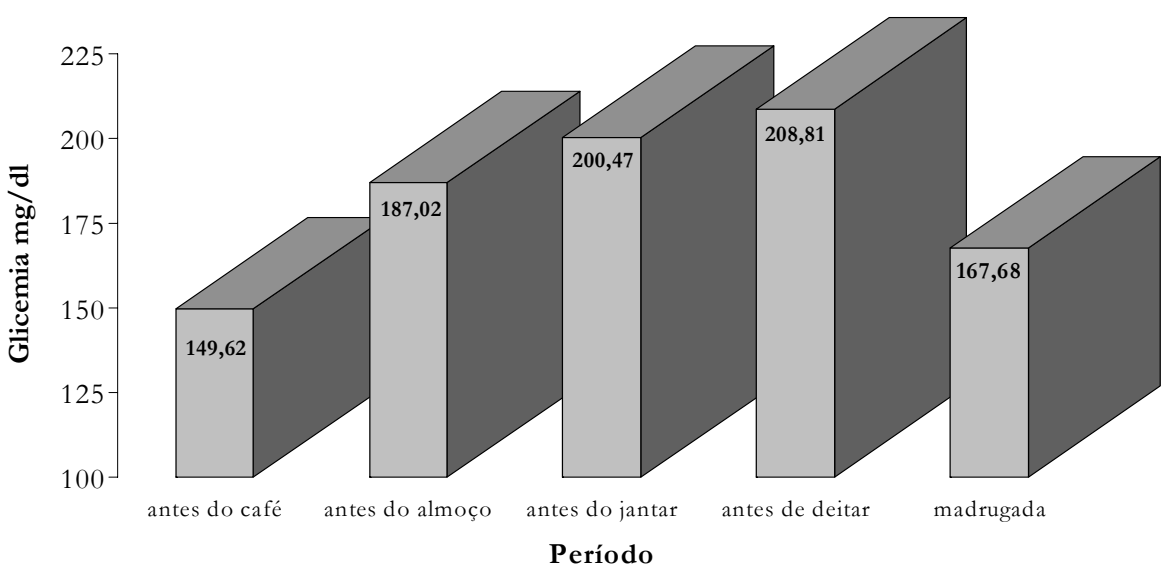

Antes do café $6,87 \%$, antes do almoço 3,83\%, antes do jantar 11,37\%, antes de deitar 30,50\% e na madrugada 19,28\%, acima do satisfatório

Gráfico 1 - Média das glicemias realizadas nos diferentes períodos. São Paulo, 1999

O gráfico 1 mostra a média das glicemias realizadas nos períodos preconizados para a realização dos controles. Pode-se observar que em todos estes períodos as médias glicêmicas foram maiores do que os índices considerados satisfatórios para cada horário.

A média das glicemias de antes do café, antes do almoço, antes do jantar, antes de deitar e madrugada, ultrapassaram os limites superiores satisfatórios em $6,87 \%, 3,83 \%$, $11,37 \%, 30,50 \%$ e $19,28 \%$ respectivamente. Estes dados mostram o maior descontrole glicêmico nos horários de antes de deitar, pela madrugada e antes do jantar, falando a favor da necessidade de mudanças nos esquemas insulinoterápicos vigentes, introduzindo maiores quantidades de insulina regular antes do jantar, maiores quantidades de insulina de ação intermediária antes do café e a terceira dose de insulina de ação intermediária antes de deitar.

As justificativas até aqui apresentadas, para a ocorrência dos descontroles glicêmicos, em todos os horários, estiveram relacionadas à inadequação entre a insulinoterapia, ingestão alimentar e as atividades físicas. Entretanto, esta pretendida adequação é extremamente difícil na vida diária do paciente diabético tendo em vista que, o impacto do diabetes e seu tratamento é único para cada indivíduo assim como suas respostas à terapêutica estabelecida e que, as características individuais e as diferentes fases da vida, influenciam não somente o curso da doença como também a adesão ao tratamento ${ }^{(5,16-18)}$. A grande demanda de comportamentos de autocuidado para o controle da doença exigem muito esforço de todas as pessoas en- volvidas com o tratamento do diabetes, principalmente no sentido de educar para o autocuidado. O desenvolvimento destes comportamentos de autocuidado é influenciado por inúmeros fatores psicossociais como crenças em saúde, locus de controle, habilidade para o enfrentamento de situações, experiências anteriores, distúrbios afetivos, conflitos familiares, maturidade cognitiva, suporte para o tratamento, bem estar emocional e prontidão para mudanças, entre outros fatores $^{(17-19)}$.

\section{Ajustes insulinoterápicos com base nos perfis glicêmicos e controle glicêmico}

Os esquemas regulares de monitorização domiciliares da glicemia propostos neste estudo, foram incorporados à vida diária dos pacientes, sem grandes dificuldades. Dos 3587 testes sangüíneos propostos, por meio de prescrições quinzenais, 3259 (90,85\%) foram realizados. Durante o desenvolvimento do estudo, a constatação da utilidade dos resultados dos testes no direcionamento dos ajustes terapêuticos, pelos pacientes e familiares, o fornecimento gratuito das tiras reagentes e o suporte dado pela equipe foram os fatores que contribuíram para a adesão à monitorização.

Os encontros educativos possibilitaram a discussão grupal da interação entre estes perfis com os picos de ação da insulina, dieta e atividade física. Desta forma a monitorização favoreceu o processo educativo voltado para o enfrentamento e resolução dos problemas práticos e reais da vida diária de cada um. $\mathrm{O}$ conhecimento advindo deste tipo de experiência confere segurança e autonomia para a 
tomada de decisões e quanto maior a participação do paciente e familiar neste processo maior é adesão ao autocuidado ${ }^{(1,3,20)}$.

Os esquemas de monitorização propostos neste estudo viabilizaram a construção de perfis individuais que subsidiaram os ajustes nas doses de insulina NPH e regular matutinais e vespertinas. Foram implemen-tados 95 (46,57\%) ajustes de dose na insulina NPH matutina sendo, 76 ajustes para mais e 19 para menos; 11 $(5,42 \%)$ ajustes de dose na insulina regular matutina sendo, 8 para mais e 3 para menos; 44 $(21,78 \%)$ ajustes de dose de insulina NPH de antes do jantar sendo, 26 para menos e 18 para mais e $22(10,84 \%)$ ajustes realizados nas doses de insulina regular feitas antes do jantar, sendo 15 para mais e 7 para menos.

A análise dos ajustes das doses de insulina NPH(antes do café) e R(antes do jantar) permite concluir que nas duas situações houve predominância dos ajustes para mais. Uma relação entre estes ajustes e a média dos perfis glicêmicos dos pacientes, nos diferentes horários e ao longo do estudo, possibilita avaliar que os ajustes preconizados tiveram a correta intenção de reduzir os níveis glicêmicos mais insatisfatórios ou seja, dos períodos antes do jantar e antes de deitar. Desta forma, os aumentos nas doses de insulina NPH matutina, a qual tem seu pico de ação predominantemente no período da tarde, foram realizados na tentativa de reduzir a glicemia de antes do jantar que apresentouse $11,37 \%$ acima do limite superior satisfatório.Os aumentos nas doses de insulina regular vespertina, a qual tem seu pico de ação 2 a 4 horas após o jantar, foram implementados para redução da glicemia de antes de deitar que apresentou-se 30,5\% acima do limite superior satisfatório (gráfico 1).

Embora a média das glicemias da madrugada e de antes do café tenham ultrapassado os limites considerados satisfatórios em $19,28 \%$ e $6,87 \%$ respectivamente, os ajustes necessários nas doses da insulina NPH vespertina foram mais cautelosas tendo em vista a possibilidade de maior ocorrência de hipoglicemias na madrugada (gráfico 1).

A média das glicemias de antes do almoço, mesmo tendo ultrapassado os limites satisfatórios em 3,83\%, foi a melhor de todos os horários. Isto se explica pelo fato de que, em estando o paciente acordado, os sinais de hipoglicemia são facilmente percebidos, o que permite o uso de doses maiores de insulina regular antes do café e também, pelo menor teor de carboidratos contido nos lanches anteriores ao almoço (gráfico 1).

A comparação entre os esquemas insulinoterápicos iniciais e finais ao estudo, mostraram que o esquema de 1 única dose diária foi mantido em $29,41 \%$ dos pacientes, o esquema de 2 doses diárias reduziu de 70,58\% para $58,82 \%$ e o esquema de 3 doses diárias foi instituído para $11,76 \%$ dos pacientes. Com relação a quantidade de insulina administrada por $\mathrm{Kg}$ de peso corporal, o percentual de pacientes, que recebia menos de $1 \mathrm{U} / \mathrm{Kg} / \mathrm{dia}$, passou de $76,47 \%$ para $52,94 \%$ e o percentual que recebia mais do que $1 \mathrm{U} / \mathrm{Kg} /$ dia passou de $23,52 \%$ para 47,06\%. Assim sendo, os pacientes passaram a tomar mais insulina com o decorrer do estudo.

Com relação ao controle metabólico, observou-se que houve uma melhora significativa do controle metabólico nos parâmetros de controle(HbA1c de $11,99 \%$ para $9,44 \%$, $\mathrm{p}<0,005)$, fato este que pode ser explicado pela reestruturação nos processos de trabalho desenvolvidos pela equipe do serviço, do qual fazemos parte. A enfermagem foi introduzida neste serviço, que até o momento contava somente com a equipe médica para o atendimento dos pacientes e familiares, por meio de consulta individualizada. A partir disso, ações educativas individualizadas e informais realizadas nas consultas de enfermagem, o incentivo à aquisição de monitores de glicemia e à realização da monitorização domiciliar passaram a ser o foco das atividades de enfermagem $^{(21)}$. Entretanto, a proposta de monitorização do presente estudo, que teve como objetivo fundamental usar sistematicamente os resultados dos testes domiciliares no plano terapêutico dos ajustes, visando a melhoria do controle metabólico, não evidenciou esta melhoria, embora tenha mantido os níveis de HbA1c em patamares de $10 . \%$. O teste de análise de variância para medidas repetidas mostrou que não houve diferença estatisticamente significativa na média das $\mathrm{HbA} 1 \mathrm{c}$ antes e após as intervenções implementadas $(p>0,05)$.

\section{CONSIDERAÇÕES FINAIS}

Os perfis glicêmicos possibilitaram ajustes insulinoterápicos com maior segurança entretanto, a idéia de que o uso sistemático dos resultados dos testes domiciliares

sangüíneos prescritos como parte do proces-
Caracterização dos perfis glicêmicos domiciliares como estratégia para os ajustes insulinoterápicos em pacientes com diabetes mellitus do tipo 1 
Sonia Aurora Alves Grossi Tamara Iwanow Cianciarullo Thaís Della Manna

so educativo e do planejamento terapêutico quinzenal, conforme o preconizado, pudesse melhorar ainda mais os níveis glicêmicos dos pacientes, não foi confirmada por meio deste trabalho.

Considerando que os participantes deste estudo e seus familiares, provavelmente, aumentaram seus conhecimentos sobre diabetes, é intenção da equipe reunir estes pacientes para uma proposta, ainda que não a ideal, de intensificação da monitorização sangüínea, associada a protocolos de ajustes nas doses diárias de insulina, a partir dos resultados obtidos nos testes glicêmicos. Este redirecio-

\section{REFERÊNCIAS}

(1) Loman D, Galgani C. Monitoring diabetic children's blood glucose levels at home. $\mathrm{MCN}$ Am J Matern Child Nurs 1996; 9(3):192 - 6.

(2)Geffner ME, Kaplan SA, Lippe BM, Scott ML. Self-monitoring of blood glucose levels and intensified insulin therapy: acceptability and efficacy in childhood diabetes. JAMA 1983; 249(21): $2913-6$

(3)Daneman D, Siminerio L, Transue D, Betschart J, Drash A, Becker D. The role of self-monitoring of blood glucose in the routine management of children with insulin-dependent diabetes mellitus. Diabetes Care 1985; 8(1):1 - 4 .

(4)Carney RM. Schechter K, Homa M, Levandoski L, White N, Santiago J. The effects of blood glucose testing versus urine sugar testing on the metabolic control of insulin-dependent diabetic children. Diabetes Care 1983; 6(4): 378 -80.

(5)Wolfsdorf JI, Anderson BJ, Pasquarello C. Treatment of the child with diabetes. In: Kahn C R, Weir G C, editors. Joslin's diabetes mellitus. $13^{\text {a }}$.ed. Philadelphia: Lea \& Febiger; 1994; p.530-51.

(6)Diabetes Control and Complications Trial Research Group. The effect of intensive treatment of diabetes on the development and progression of long-term complications in insulin-dependent diabetes mellitus. N Engl J Med 1993; 329(14):977 - 86.

(7)Hoogwerf BJ, Brouhard BH. Glicemic control and complications of diabetes mellitus: pratical implications of Diabetes Control and Complications Trial. Cleve Cli J Med 1994; 61(1): $34-7$.

(8)Glycated hemoglobin. Abbot Park:Abbott Laboratories, 1994.

(9)American Diabetes Association. Standards of medical care for patients with diabetes mellitus. Diabetes Care1997; 20 (Suppl 1): 5 - 13. namento, é sentido pela equipe como algo possível de ser realizado diante das limitações individuais para o autocontrole e das deficiências do serviço.

É possível pensar que os participantes deste estudo, possam continuar a ser estimulados, por meio de abordagens educacionais compreensivas individualizadas ou grupais, para as mudanças desejadas de comportamentos e envolvidos em um treinamento mais avançado objetivando os auto-ajustes diários a partir da disponibilidade na realização de maior número de monitorizações.
(10)American Diabetes Association. Standards of medical care for patients with diabetes mellitus. Diabetes Care 2000; 23 (Suppl 1): 32 - 42.

(11)American Diabetes Association. Medical management of type 1 diabetes. $3^{\mathrm{a}} \mathrm{ed}$. Alexandria, 1998a. Routine management: tools; p. 55 - 83.

(12)Rosenzweig JL. Principles of insulin therapy. In: Kahn C R, Weir G C, editors. Joslin's diabetes mellitus. $13^{\text {a }}$.ed. Philadelphia: Lea \& Febiger; 1994. p. 460 - 88.

(13)Damiani D. Qual a melhor maneira de tratar o diabetes mellitus. In: Setian N, Damiani D, Dichtchkenian V, editores. Diabetes mellitus na criança e no adolescente: encarando o desafio. São Paulo: Sarvier, 1995. p. 57 - 76.

(14)American Diabetes Association. Medical management of type 1 diabetes. $3^{\mathrm{a} e d}$ Alexandria, 1998b. Hipoglycemia; p.134 - 44:

(15)Kitabchi AE. Diabetes ketoacidoses and hyperglycemic, hyperosmolar nonketotic state. In: Kahn C R, Weir G C, editors. Joslin's diabetes mellitus. $13^{\text {a }}$.ed. Philadelphia: Lea \& Febiger; 1994. p. 738 - 70.

(16)Betschart J. Diabetes and the life cycle. In: American association of Diabetes Educators. a core curriculum of diabetes education. $3^{\text {a }}$.ed. Chicago, 1998. p. 537 - 67.

(17)Jacobson AM, Hansen ST, Lavon P, Willett JB, Cole CF, Wolfsdorf et al.. Family environment and glycemic control:a four year prospective study of children and adolescents with insulin-dependent diabetesmellitus. Psychosom. Med 1994; 56(5):401-9.

(18)Anderson B, Ho J, Brackettj, Finkelstein D, Laffel L. Parental involvement in diabetes management tasks: relationships to blood glucose monitoring adherence and metabolic control in young adolescents with insulindependent diabetes mellitus. J Pediatr 1997; 130(2):257-65. 
(19)Rubin RR. Psychosocial assessment. In: American Association of Diabetes Educators. A core curriculum for diabetes education. $3^{\mathrm{a}}$.ed. Chicago, 1998. p. 87 - 118.

(20)Maldonato A, Bloise D, Ceci M, Fraticelli E, Fallucca F. Diabetes mellitus: lessons from patient education. Patient Educ Couns 1995; 26(1/3): $57-66$.
(21)Grossi SAA, Della Manna T, Andrade RSF, Ferrari LAP, Kuperman H. et al. Influência de ações educativas e da monitorização domiciliar da glicemia no controle metabólico de crianças e adolescentes de baixa renda portadores de diabetes mellitus do tipo 1. Arq Bras Endocrinol Metabol 1998; 42 (5): 228.
Caracterização dos perfis glicêmicos domiciliares como estratégia para os ajustes insulinoterápicos em pacientes com diabetes mellitus do tipo 1

Recebido: 03/05/2002

Instrumento 4

ANEXO

\begin{tabular}{|l|l|l|}
\hline I. Identificação & \\
\hline Nome: & Registro: & Data: \\
\hline Cuidador(es): & \multicolumn{2}{l|}{} \\
\hline
\end{tabular}

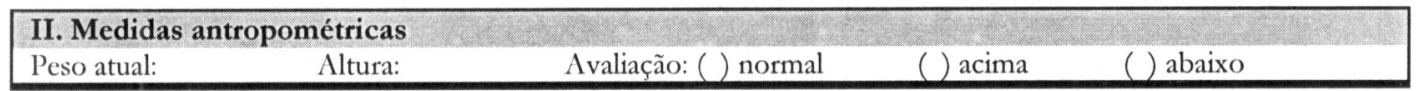

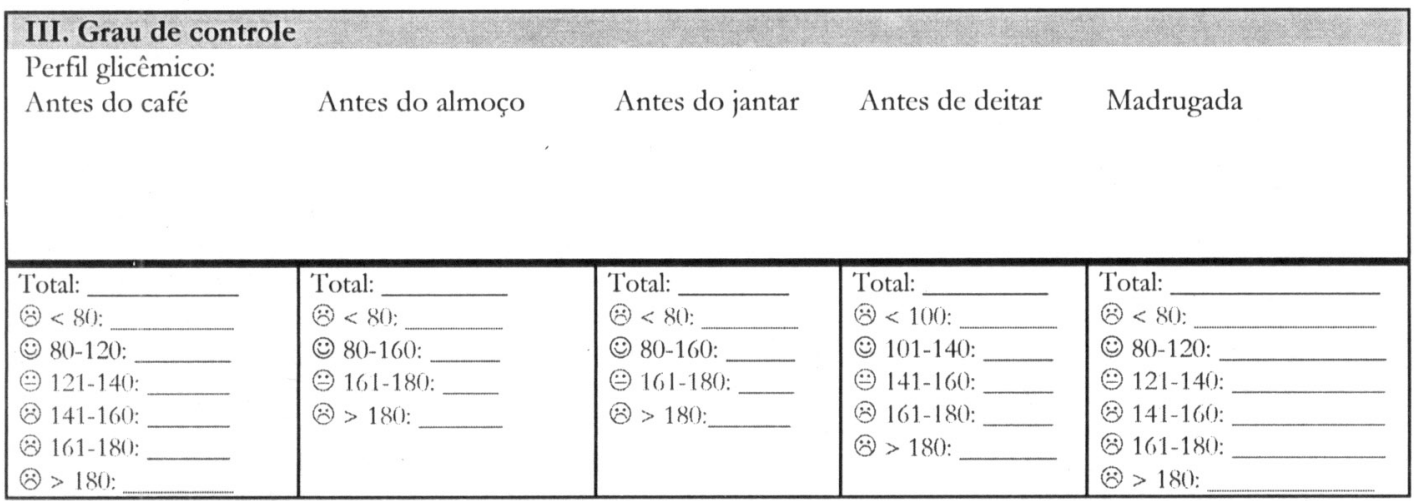

Legenda: :) bom, :- aceitável, : ruim (American Diabetes Association, 1997)

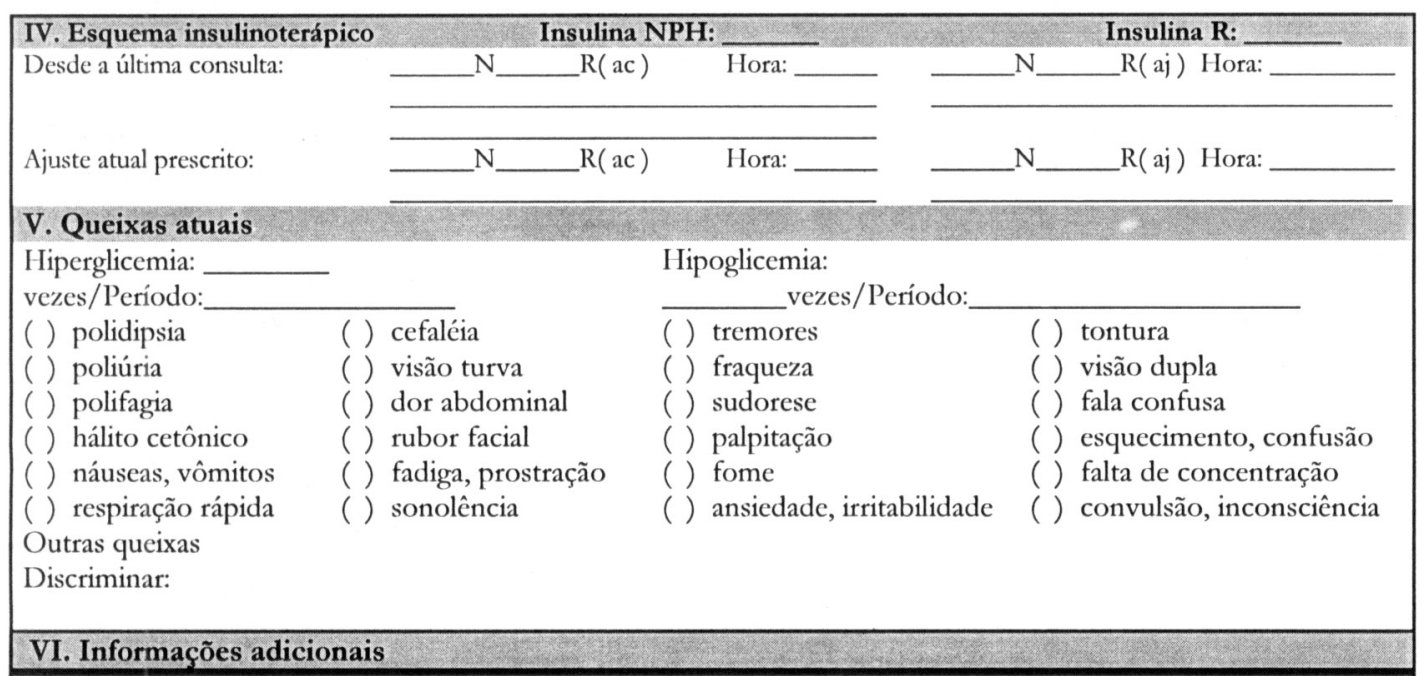

